\title{
Congenital Facial Paralysis
}

\section{Vijaya Krishna Varanasi*}

Washington State University, USA

Open Access has become a passionately discussed topic all over the world. Open Access can be defined as a 'global public good': an instrument to stimulate the growth and quality of global science, as well as an instrument for realizing the rights to share in scientific advancement and its benefits, to education and to information (articles 27, 26 and 19 of the Universal Declaration of Human Rights respectively) [1]. Open Access (OA) journals such as 'Anaplastology' from OMICS Publishing Group allow researchers to maximize dissemination of their work by reaching the largest possible readership. Articles published in open access format are more likely to be cited than those that are not [2]. Due to this reason impact factor of open access journals is on the rise for the last couple of years [3]. Researchers in developing nations are more likely to access information published by OA journals. OMICS Publishing Group strongly supports the open access initiative and all articles published by OMICS Group are freely accessible to everyone immediately after publication. Some of the special features of OMICS group journals include digital formatting, audio listening, language translation and ability to share views on articles via social networking. I strongly believe that latest research results in congenital facial paralysis and other related topics should be made available to the medical fraternity and common man so that they are aware of the various treatment options available to rectify the problem. The only way this is possible is through the medium of Open Access and through journals such as 'Anaplastology'. The field of Anaplastology is an art and science dealing with the prosthetic rehabilitation of an absent, disfigured, or malformed anatomically critical location of the face or body. 'Anaplastology' is an open access scientific international journal which is peer-reviewed and publishes the most exciting research related to the development of Anaplastology and their diagnostic applications.

Congenital facial paralysis is present right from the time of birth and its frequency is about two out of every 1000 newborns. About $8-14 \%$ of all pediatric cases of facial paralysis fall under the category of congenital type [4]. The most common unilateral syndromic condition associated with facial paralysis is Hemifacial microsomia. Möbius syndrome on the other hand is an example of a facial paralysis due to developmental cause with a frequency of 1 per 50,000 births [5]. Common problems faced by the newborns suffering from congenital facial paralysis are difficulty with nursing and incomplete eye closure. This condition needs to be addressed at an early stage of life, otherwise the child's future speech might be affected, which in turn might affect their self esteem. The symptoms of congenital facial paralysis in children tend be to more conspicuous with increasing age.

A traumatic injury or developmental deformities of the brain can affect the functioning of the cranial nerves VI and VII leading to congenital facial paralysis. These nerves control movement of the eyes and various facial expressions. The cranial nerves originate in the brain and innervate the muscles on both sides of the face creating facial expressions. The cranial nerves are also important to protect the eyes, pronounce words properly, and keep food and liquids in the mouth.

The most frequent cause of unilateral congenital facial palsy is birth trauma due to a difficult delivery. There is no need of surgery in case of congenital facial nerve paralysis occurring due to trauma because of its self healing nature [6]. But surgery is recommended for patients with developmental facial paralysis and is usually delayed until later stages of life.
Professionals dealing with developmental and unresolved traumatic facial paralysis recommend initial surgery during preschool to overcome psychosocial problems associated with a physical abnormality. But there are others who recommend surgery at adolescence when facial growth is mature and the child is able to understand the risks [7]. Recently, a study was done to systematically describe the various stigmata and anomalies associated with developmental facial paralysis [8]. The study found that developmental facial paralysis correlated to the presence of amblyopia (lazy eye), underdeveloped facial nerve (hypoplasia), lower alar atresia, and dermatological changes. In part two of the study by the same authors on outcomes of reconstructive surgery [9], there was an improvement in the outcome scores for all patients (preoperatively, 2.44; 2 years postoperatively, 3.66; final, 4.11). There was greater improvement in eye closure, smile, and depressor function in children relative to adults. The authors concluded that screening, diagnosis, and treatment for developmental facial paralysis done at an early stage of life improves the physical and emotional wellbeing of children, facilitating merger with their peers. It is also critical to distinguish between developmental and traumatic facial paralysis observed at birth for prognostic and medico legal reasons. The need of the hour is to better understand developmental facial paralysis in children and in facially disfigured individuals in order to facilitate their rehabilitation and blending among peers.

\section{References}

1. UNESCO (2011) A Global Perspective on Open Access.

2. MacCallum CJ, Parthasarathy $H$ (2006) Open access increases citation rate. PLoS Biol 4: 176.

3. http://www.biomedcentral.com/download/advocacy/bmcfactsheet2.pdf

4. Evans AK, Licameli G, Brietzke S, Whittemore K, Kenna M (2005) Pediatric facial nerve paralysis: patients, management and outcomes. Int J Pediatr Otorhinolaryngol 69: 1521-1528.

5. Mchugh HE (1963) Facial Paralysis in birth injury and skull fractures. Arch Otolaryngol 78: 443-455

6. Bergman I, May M, Wessel HB, Stool SE (1986) Management of facial palsy caused by birth trauma. Laryngoscope 96: 381-384.

7. Shapiro NL, Cunningham MJ, Parikh SR, Eavey RD, Cheney ML (1996) Congenital unilateral facial paralysis. Pediatrics 97: 261-264.

8. Terzis JK, Anesti K (2011) Experience with developmental facial paralysis: Part I. Diagnosis and associated stigmata. Plast Reconstr Surg 128: 488-497.

9. Terzis JK, Anesti K (2012) Experience with developmental facial paralysis: Part II. Outcomes of reconstruction. Plast Reconstr Surg 129: 66-80

*Corresponding author: Vijaya Krishna Varanasi, Washington State University, USA, E-mail: vijaya.varanasi@gmail.com

Received January 30, 2012; Accepted January 30, 2012; Published February 05, 2012

Citation: Vijaya Krishna V (2012) Congenital Facial Paralysis. Anaplastology 1:e101. doi: 10.4172/2161-1173.1000e101

Copyright: () 2012 Vijaya Krishna V, et al. This is an open-access article distributed under the terms of the Creative Commons Attribution License, which permits unrestricted use, distribution, and reproduction in any medium, provided the original author and source are credited. 